\title{
Scandinavian Journal of Gastroenterology
}

\section{Protein induced by vitamin $\mathrm{K}$ absence or antagonist-II (PIVKA-II) specifically increased in Italian hepatocellular carcinoma patients}

\section{Valentina Viggiani, Sara Palombi, Giuseppina Gennarini, Gabriella D’Ettorre, Corrado De Vito, Antonio Angeloni, Luigi Frati \& Emanuela Anastasi}

To cite this article: Valentina Viggiani, Sara Palombi, Giuseppina Gennarini, Gabriella D'Ettorre, Corrado De Vito, Antonio Angeloni, Luigi Frati \& Emanuela Anastasi (2016): Protein induced by vitamin $\mathrm{K}$ absence or antagonist-II (PIVKA-II) specifically increased in Italian hepatocellular carcinoma patients, Scandinavian Journal of Gastroenterology, DOI: 10.1080/00365521.2016.1183705

To link to this article: http://dx.doi.org/10.1080/00365521.2016.1183705

Published online: 26 May 2016.

Submit your article to this journal 지

ai

View related articles $\sqsubset$

View Crossmark data $\nearrow$ 


\title{
Protein induced by vitamin K absence or antagonist-II (PIVKA-II) specifically increased in Italian hepatocellular carcinoma patients
}

\author{
Valentina Viggiani ${ }^{a}$, Sara Palombi ${ }^{a}$, Giuseppina Gennarini ${ }^{a}$, Gabriella D’Ettorre $^{\mathrm{b}}$, Corrado De Vito $^{\mathrm{b}}$, \\ Antonio Angeloni ${ }^{a}$, Luigi Frati ${ }^{\mathrm{a}}$ and Emanuela Anastasi ${ }^{\mathrm{a}}$ \\ aDepartment of Molecular Medicine, "Sapienza" University of Rome, Roma, Italy; 'Department of Public Health and Infectious Diseases, \\ "Sapienza" University of Rome, Roma, Italy
}

\begin{abstract}
Objective: As a marker for Hepatocellular Carcinoma (HCC), Protein Induced by Vitamin K Absence II (PIVKA-II) seems to be superior to alpha fetoprotein (AFP). To better characterize the role of PIVKA-II, both AFP and PIVKA-II have been measured in Italian patients with diagnosis of HCC compared with patients affected by non-oncological liver pathologies.

Materials and methods: Sixty serum samples from patients with HCC, 60 samples from patients with benign liver disease and 60 samples obtained from healthy blood donors were included in the study. PIVKA-II and AFP were measured by LUMIPULSE ${ }^{\circledR}$ G1200 (Fujirebio-Europe, Belgium). We considered as PIVKA-II cutoff $70 \mathrm{mAU} / \mathrm{ml}$ (mean +3SD) of the values observed in healthy subjects.

Results: The evaluation of PIVKA-II showed a positivity of $70 \%$ in patients with HCC and $5 \%$ in patients with benign diseases $(p<0.0001)$ whereas high levels of AFP were observed in $55 \%$ of HCC patients and in $47 \%$ of patients with benign diseases. The combined Receiver Operating Characteristic (ROC) analysis of the two analytes revealed a higher sensitivity (75\%) compared to those observed for the individual biomarkers. In conclusion, we demonstrate that as a marker for HCC, PIVKA-II is more specific for HCC and less prone to elevation during chronic liver diseases.

Conclusions: The combination of the two biomarkers, evaluated by the ROC analysis, improved the specificity compared to a single marker. These data suggest that the combined analysis of the two markers could be a useful tool in clinical practice.
\end{abstract}

\section{ARTICLE HISTORY}

Received 19 February 2016

Revised 21 April 2016

Accepted 22 April 2016

Published online 25 May 2016

\section{KEYWORDS}

AFP; benign liver diseases; hepatocellular carcinoma; PIVKA-II; tumor marker

\section{Introduction}

Worldwide, the hepatocellular carcinoma (HCC) is the eighth most common type of solid cancer and the third most common cause of cancer-related deaths. The deaths attributed to this form of cancer are a million a year with a mortality around 94\%.[1]

For the diagnosis of HCC, alpha fetoprotein (AFP) is the most commonly used biomarker. Many studies suggest that the persistent high values of AFP should be evaluated as a risk factor for developing HCC.

In virtue of this, AFP has been considered for a long time as a good biomarker to identify subjects at high risk for HCC.[2-4]

However, like many other biomarkers, AFP is not tumorspecific, but is produced and released from the liver tissue in physiological conditions and in the presence of various oncological and non-oncological diseases. It is well known that AFP may occasionally be produced in significant quantities even by other different organs or tissues than the liver.[5-7]

The AFP diagnostic sensitivity for early-stage HCC is only $48 \%$,[8] and therefore, according to the recent guidelines, AFP is not recommended for HCC surveillance an early diagnosis.[9]
Because of the poor sensitivity and specificity of AFP, in the recent years, research has focused on the identification of new biomarkers that can provide higher sensitivities and specificities.[10]

Protein Induced by Vitamin K Absence II (PIVKA-II, desgamma carboxyprothrombin), is a biomarker identified by Liebmann some years ago.[11] More recently, PIVKA-II has been proposed as an emerging circulating marker in HCC. The first assays were based on the competitive radioimmunoassay principle using PIVKA-II polyclonal antibodies. Later, after the early promising results, in the last two decades, PIVKA-II has been introduced as a serological marker for HCC detection.[12,13]

It is extensively recognized that the main role of biomarkers should be as an early indicator of cancer as well as an ideal index for the differential diagnosis between benign or not benign disease. With regard to early and differential diagnosis of HCC, many studies showed a better specificity of PIVKA-II in comparison with AFP. Finally, it is important to highlight that PIVKA-II is a promising biomarker capable of following the remission of the disease and monitoring the response to therapy.[14]

To date, PIVKA-II has been mainly investigated in Asian countries, and inspite of its potential diagnostic and 
prognostic value, knowledge about PIVKA-II in Europe, and particularly in Italy, remains limited.

To better characterize the role of PIVKA-II in patients with $\mathrm{HCC}$, we measured PIVKA-II and AFP levels in a group of patients with diagnosis of HCC compared to a group of patients affected by Hepatitis C Virus (HCV), Hepatitis B Virus $(\mathrm{HBV})$ and space occupying lesion (SOL).

\section{Patients and methods}

\section{Patients}

All subjects included in the study were patients admitted to our laboratory. All patients and subjects gave their informed consent for the investigation. For this study, we analyzed 180 serum samples with following characteristics:

a. Sixty serum samples from patients with HCC (age range: 39-86; 41 males, 19 females);

b. Sixty serum samples from patients with benign liver disease: HCV, HBV and SOL (age range: 26-84, 23 males, 37 females);

c. Sixty serum samples obtained from a population of healthy blood donors (age range: 22-74, 26 males, 34 females).

\section{Sample preparation}

All sera were acquired following a standard collection protocol. Briefly, samples were collected in a Red Top Vacutainer, clotted $60-90 \mathrm{~min}$ and centrifuged for $10 \mathrm{~min}$ at $1300 \mathrm{~g}$. The serum fractions were aliquoted and stored at $-80^{\circ} \mathrm{C}$ until analysis.

\section{Biomarker assays}

The levels of PIVKA-II and AFP were measured by LUMIPULSE G1200 (Fujirebio-Europe, Belgium).[15] All assays were performed according to the manufacturers' instructions. LUMIPULSE $^{\circledR}$ G1200 (Fujirebio-Europe, Belgium) is an assay system for the quantitative measurement in serum or plasma specimens based on chemiluminescent enzyme immunoassay (CLEIA) technology by a two-step sandwich in immunoreaction cartridges (Fujirebio Europe NV, Belgium). This assay makes use of monoclonal antibody-coated beads and alkaline phosphatase (ALP)-labeled monoclonal antibody. In the first reaction, the analyte specifically binds to monoclonal antibody on the particles forming antigen-antibody immunocomplexes. In the second reaction, ALP-labeled monoclonal antibody binds to the analyte of the immunocomplexes. Then the adamantyl1,2-dioxetane phosphate (AMPPD) contained in the substrate solution is dephosphorylated by the catalysis of ALP indirectly conjugated to the particles. A luminescent signal is generated by the cleavage reaction of dephosphorylated AMPPD and reflects the amount of antigen in the sample.

According to the manufacturer's indications, expected normal values are, for AFP in a range of $1.7-7.4 \mathrm{ng} / \mathrm{mL}$ and for PIVKA-II in a range of $16-48 \mathrm{mAU} / \mathrm{mL}$.
Regarding the clinical cut-points of PIVKA-II, according to literature discordant between Japanese and American studies, we defined the cutoff on our population.

\section{Statistical analysis}

Sensitivity, specificity, positive predictive value (PPV) and negative predictive value (NPV) of each biomarker were calculated. Differences in accuracy of the two biomarkers between the HCC and the other benign pathologies were evaluated by the Chi-squared test. The diagnostic accuracy of the assays was assessed by estimation of the Receiver Operating Characteristic (ROC) curve for HCC cases versus benign liver diseases using MedCalc V 4.30 Software (Italy).

\section{Results}

Definition of normal levels in our healthy population: PIVKA-II

Sixty blood donors were included in this study. These samples were evaluated thanks to a collaboration with the Blood Bank of the Policlinico Umberto I. On the basis of these results, we considered as cutoff $70 \mathrm{mAU} / \mathrm{mL}(35.5 \pm 11.5$; mean $\pm 3 \mathrm{SD})$.

To note that none of the healthy subjects showed PIVKA-II values above the limit of positivity. We observed a highly statistically significant difference between HCC patients and patients affected by benign liver disease $(p<0.0001)$ (Figure 1).

We observed high levels of AFP in $55 \%$ of HCC patients and in $47 \%$ of patients with benign diseases, while it was $5 \%$ in the control group (Figure 2).

No statistically significant difference was observed between the patients with HCC versus those with benign disease.

The PIVKA-II specificity and sensitivity studied in the two selected groups of patients showed, by statistical analysis, that the area under the ROC curve was $0.814(95 \% \mathrm{Cl}$ 0.735-0.89).

The statistical analysis for AFP in the same two group of patients showed that the area under the ROC curve was 0.618 (95\% Cl 0.516-0.720).

The PIVKA-II ROC curve analysis showed, that the best specificity (0.90) and sensitivity (0.60) are obtained with a cutoff $47 \mathrm{mAU} / \mathrm{mL}$, with a PPV of 0.86 and a NPV of 0.69 (Figure 3).

Whereas the AFP ROC curve showed the best specificity (0.55) and sensitivity (0.55) with a cutoff of $20 \mathrm{mAU} / \mathrm{mL}$, with both PPV and NPV of 0.55 (Figure 4).

Comparison of the ROC curves of the combination of PIVKA-II and AFP resulted in the highest sensitivity (75\%) and a specificity of $61 \%$, with PPV and NPV of $62 \%$ and $70 \%$, respectively (Figure 5 ).

\section{Discussion}

$\mathrm{HCC}$ is one of the most common cancers worldwide with a high rate of mortality. Diagnostic surveillance of at-risk patients is done by ultrasound, sometimes completed with the measurement of a tumor marker. However, the accuracy 


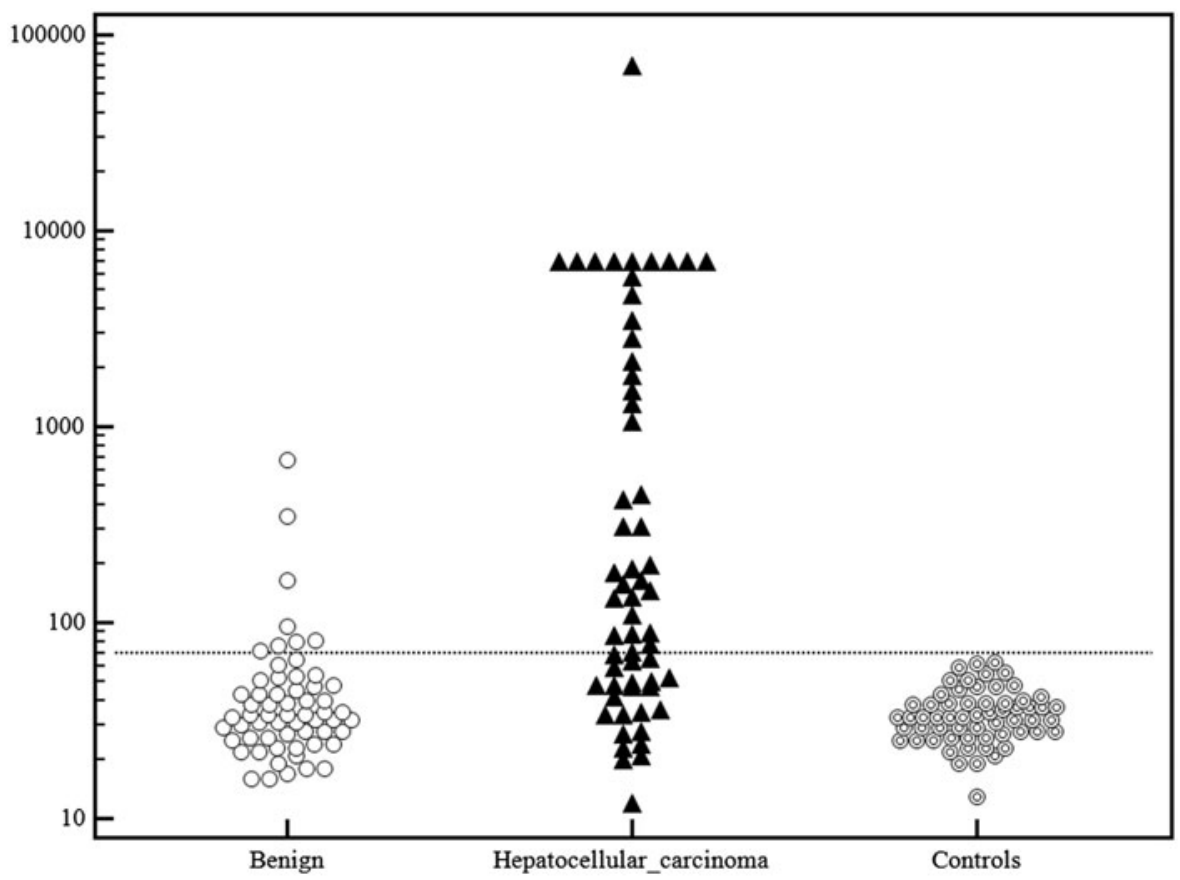

Figure 1. PIVKA-II: distribution of values of PIVKA-II in healthy subjects, patients with HCC, and benign liver disease (results are expressed as mAU $\mathrm{mL}$ ).

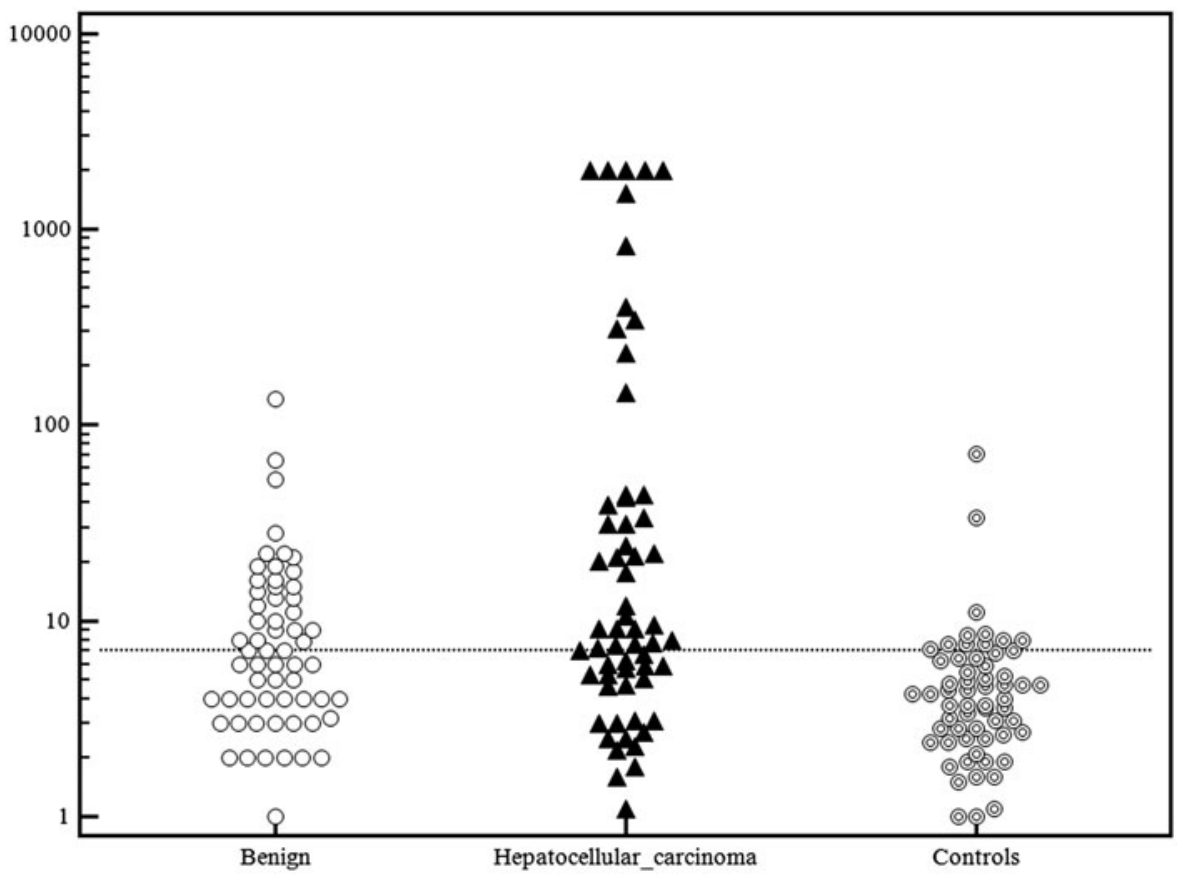

Figure 2. AFP: Distribution of values of AFP in healthy subjects, patients with HCC, and benign liver disease (results are expressed as $\mathrm{mAU} \backslash \mathrm{mL}$ ).

of ultrasound is operator-dependent and is based on his or her ability to differentiate HCC from non-neoplastic lesions such as regenerative nodules.[16]

Recently, tumor biomarkers to detect cancer have helped in surveillance of high risk patients to screen for disease and avoid wasting medical resources.[17-19]

Tumor markers are biological substances produced directly by the tumor or by non-tumor cells as a response to the presence of a tumor. They are usually detected in a solid tumor, in circulating tumor cells in peripheral blood, in lymph nodes, in bone marrow, or in other body fluids (urine, stool, ascites).[20] Thus far, more than 20 different tumor markers have been studied and characterized, but only few of them have gained a greater importance in clinical routine.

The most widely used biomarker for HCC is serum AFP. Serum AFP is commonly regarded as a supplementary parameter for diagnosis of HCC and a tool for predicting recurrence and survival. but its clinical use in the management of HCC patients is limited because AFP often is increased in other oncologic and inflammatory diseases.[21-25]

Research has been recently focusing on a new biomarker known as PIVKA-II that may provide superior utility over current markers.[22,23] 


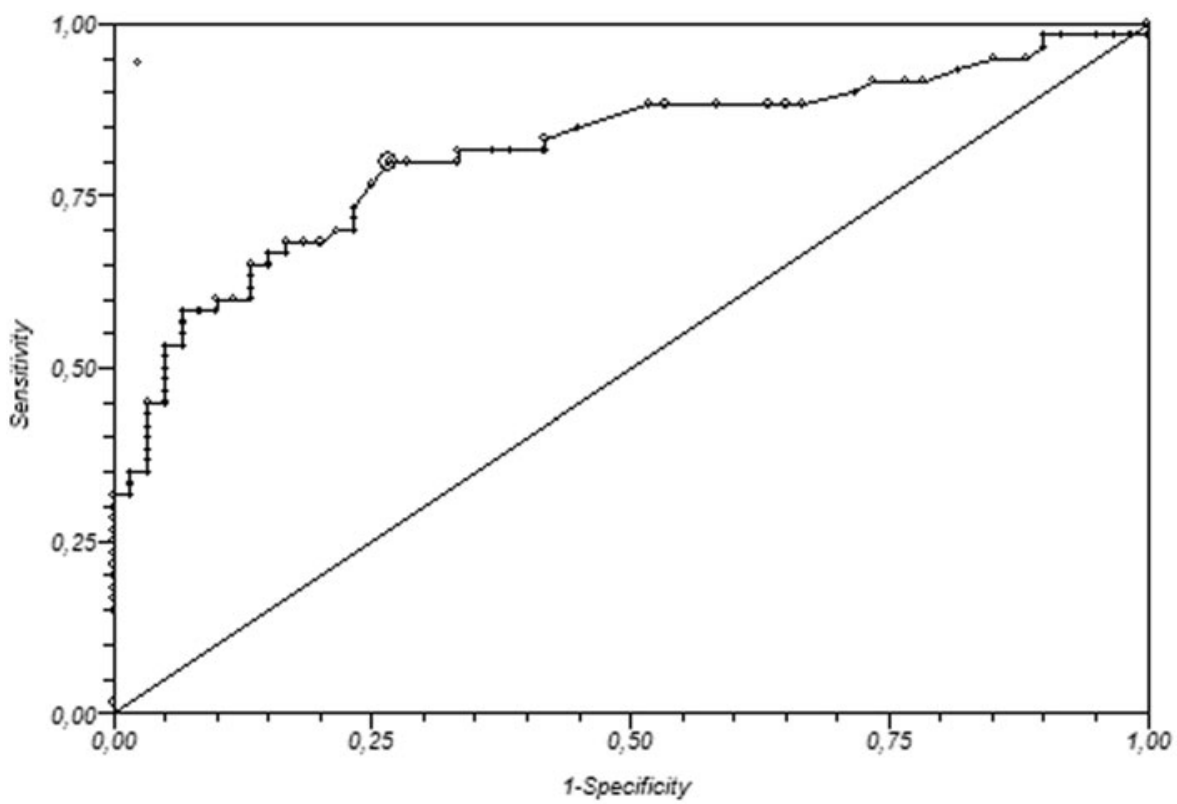

Figure 3. ROC analysis of PIVKA-II.

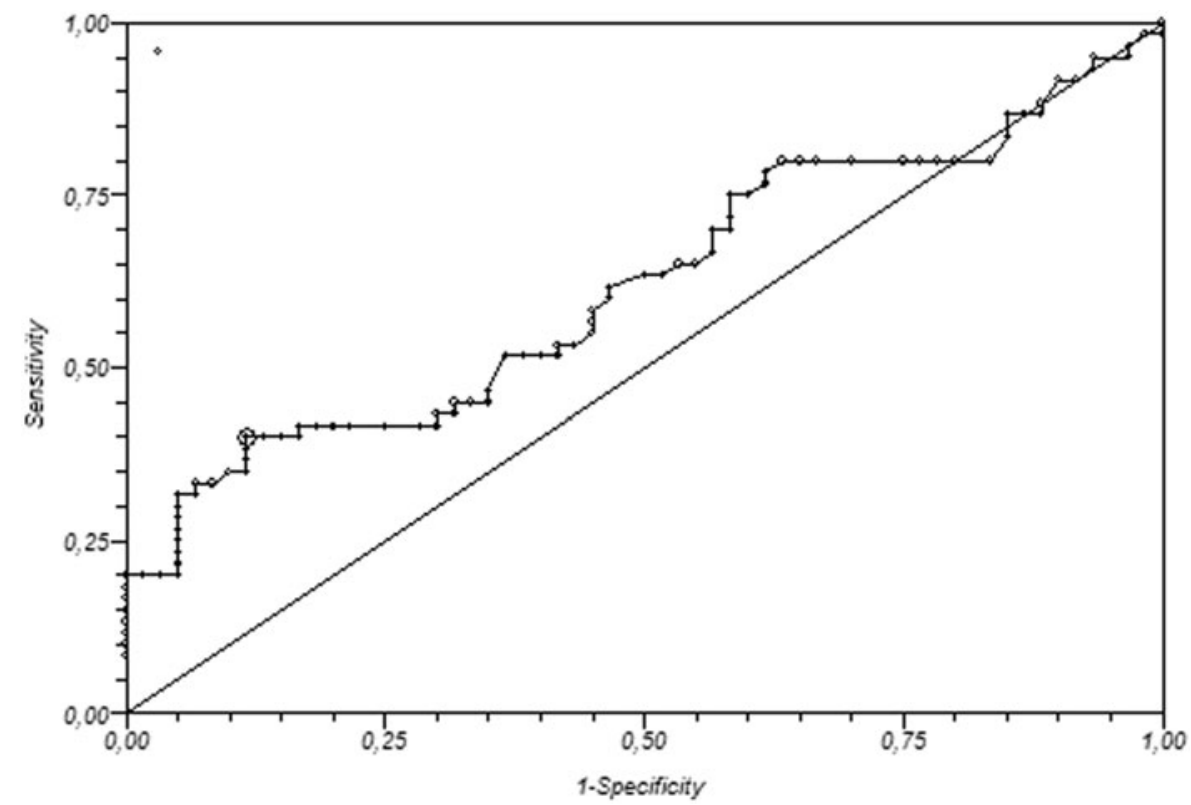

Figure 4. ROC analysis of AFP.

Since Liebman et al. demonstrated PIVKA-II to be a useful marker for HCC diagnosis, many studies have compared PIVKA-II and AFP.[24,26-28]

A PIVKA-II level of $40 \mathrm{mAU} / \mathrm{mL}$ is now commonly used as the cutoff value for diagnosis of HCC.

Many studies have been reported to evaluate the usefulness of PIVKA-II for HHC diagnosis. Regrettably the sensitivity and sensitivity described by these studies were rather different. The most important reason for these differences involves the use of different PIVKA-II cutoff in each studies. In particular, the most significant difference in marker cutoff is observed in the studies conducted on the Asian population than the American population. $[8,14]$ Since, in the literature there is disagreement regarding the cutoff to be taken into consideration, we felt it appropriate to evaluate a new cutoff on our population. On the basis of our data, we considered as cutoff a PIVKA-II level of $70 \mathrm{mAU} / \mathrm{mL}$ (mean $\pm 3 S \mathrm{SD}$ ). In particular with this cutoff we observed that PIVKA-II is positive in a high proportion (70\%) in HCC patients and in only $5 \%$ of patients with inflammatory diseases, while AFP is elevated in $55 \%$ of HCC and $47 \%$ of patients with benign diseases show fluctuating levels of AFP.

In this study, we demonstrated for the first time, in a cohort of Italian patients that PIVKA-II is more accurate than AFP in differentiating patients with HCC from those with nonmalignant chronic liver disease.

In particular, our results showed that as a marker for HCC, PIVKA-II may be superior to AFP. PIVKA-II is more specific to $\mathrm{HCC}$ and less prone to elevation during chronic liver disease. 


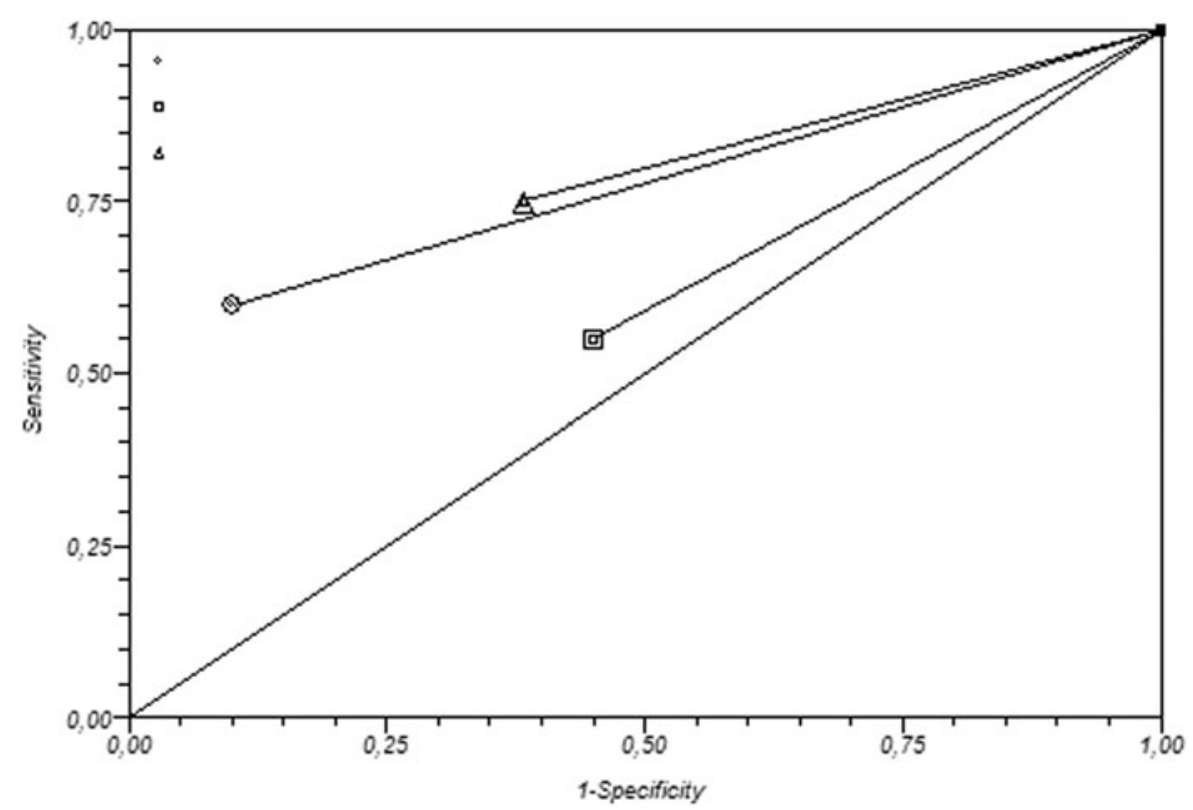

Figure 5. The combined ROC analysis of the two analytes.

The combination of two biomarkers as suggested by ROC curve analysis, indicates for the best discrimination between benign and HCC diseases to use for PIVKA-II $47 \mathrm{mAU} / \mathrm{mL}$ as cutoff. This finding is in agreement with the instruction of the manufacturer. Our next goal will be the evaluation, by prospective studies in a much larger population of patients the effective capacity to make differential and early diagnosis HCC.

This study suggests that the combined analysis of the two markers could be a useful tool in clinical practice. This could also have benefits on "health economics" by reducing the number of investigations necessary for a proper diagnosis and further clinical management of patients with HCC.

A recent study that compared AFP against PIVKA-II, demonstrated that AFP, the best prognostic single marker for the diagnosis of HCC is in disagreement with our results.[29]

Interestingly, this study has used PIVKA-II ELISA KIT technique that in comparison with PIVKA-II chemiluminescent enzyme immunoassay (CLEIA) technology shows less sensitivity and in general inferior performances.

In addition a high percentage of HCC patients enrolled in this study were infected with HBV virus, nevertheless some researchers suggests that PIVKA-II provides substantial added value to the HCC with non-viral etiologies.[30]

Although a high percentage of positivity for PIVKA-II was found among patients suffering from HCC and a low positivity percentage in other pathologies, it was however of interest to see that seven patients affected by other pathologies were also positive for PIVKA-II.

This finding is in agreement with other studies that show occasionally aberrant elevation of PIVKA-II in patients with alcoholic cirrhosis, obstructive jaundice or vitamin $\mathrm{K}$ deficiency.[31]

Our results are very encouraging but there are several important limitations in our study. The sample size was small and the tumor staging was not well known and for these reasons we consider it as a preliminary study. More large scale and multicenter studies are needed to assess the clinical usefulness of PIVKA-II in the diagnosis and clinical management of HCC patients.

\section{Acknowledgements}

This study is founded by The University of Rome "Sapienza". We are thankful to Barbara Colaprisca and Silvestra Tudini for their technical assistance.

\section{Disclosure statement}

The authors report no conflicts of interest. The authors alone are responsible for the content and writing of this article.

\section{References}

[1] Siegel R, Naishadham D, Jemal A. Cancer statistics, 2013. CA Cancer J Clin. 2013;63:11-30.

[2] Taketa K. Alpha-fetoprotein: reevaluation in hepatology. Hepatology. 1990;12:1420-1432

[3] Tsukuma H, Hiyama T, Tanaka S, et al. Risk factors for hepatocellular carcinoma among patients with chronic liver disease. N Engl J Med. 1993;25:1797-1801.

[4] Forner A, Llovet JM, Bruix J. Hepatocellular carcinoma. Lancet. 2012;31:1245-1255.

[5] Tomasi TB Jr, Structure and function of alpha-fetoprotein. Annu Rev Med. 1977;28:453-465.

[6] Nunez EA. Biological role of alpha-fetoprotein in the endocrinological field: data and hypotheses. Tumour Biol. 1994;15:63-72.

[7] Mizejewski GJ, MacColl R. Alpha-fetoprotein growth inhibitory peptides: potential leads for cancer therapeutics. Mol Cancer Ther. 2003;2:1243-1255.

[8] Li C, Zhang Z, Zhang P, et al. Diagnostic accuracy of des-gammacarboxy prothrombin versus $\alpha$-fetoprotein for hepatocellular carcinoma: a systematic review. Hepatol Res. 2014;44:E11-E25.

[9] European Association for the study of the liver, European Organization for Research and Treatment of cancer. EASL-EORT clinical practice guidelines: management of hepacellular carcinoma. J Hepatol. 2012;56:908-943. 
[10] Bae JS, Park SJ, Park KB, et al. Acute exacerbation of hepatitis in liver cirrhosis with very high levels of alpha-fetoprotein but no occurrence of hepatocellular carcinoma. Korean J Intern Med. 2005;20:80-85.

[11] Liebman HA, Furie BC, Tong MJ, et al. Des-gamma-carboxy (abnormal) prothrombin as a serum marker of primary hepatocellular carcinoma. N Engl J Med. 1984;310:1427-1431.

[12] Lok AS, Sterling RK, Everhart JE, et al. HALT-C Trial Group. Desgamma-carboxy prothrombin and alpha-fetoprotein as biomarkers for the early detection of hepatocellular carcinoma. Gastroenterology. 2010;138:493-502.

[13] Song P, Gao J, Inagaki Y, et al. Biomarkers: evaluation of screening for and early diagnosis of hepatocellular carcinoma in Japan and china. Liver Cancer. 2013;2:31-39.

[14] Koike $Y$, Shiratori $Y$, Sato $S$, et al. Des-gamma-carboxy prothrombin as a useful predisposing factor for the development of portal venous invasion in patients with hepatocellular carcinoma: a prospective analysis of 227 patients. Cancer. 2001;91:561-569.

[15] Falzarano R, Viggiani V, Michienzi S, et al. Evaluation of a CLEIA automated assay system for the detection of a panel of tumor markers. Tumour Biol. 2013;34:3093-3100.

[16] Sheu JC, Sung JL, Chen DS, et al. Early detection of hepatocellular carcinoma by real-time ultrasonography. A prospective study. Cancer. 1985;56:660-666.

[17] Sturgeon C. Practice guidelines for tumor marker use in the clinic. Clin Chem. 2002;48:1151-1159.

[18] Huang J, Zeng Y. Current clinical uses of the biomarkers for hepatocellular carcinoma. Drug Discov Ther. 2014;8:98-99.

[19] Beale G, Chattopadhyay D, Gray J, et al. AFP, PIVKA-II, GP3, SCCA-1 and follisatin as surveillance biomarkers for hepatocellular cancer in non-alcoholic and alcoholic fatty liver disease. BMC Cancer. 2008;8:200. doi: 10.1186/1471-2407-8-200.

[20] Duffy MJ, Sturgeon CM, Sölétormos G, et al. Validation of new cancer biomarkers: a position statement from the European group on tumor markers. Clin Chem. 2015;61:809-820.

[21] Bruix J, Sherman M. Management of hepatocellular carcinoma heapatology. Hepatology. 2005;42:1208-1236.
[22] Bae $\mathrm{HM}$, Lee $\mathrm{JH}$, Yoon $\mathrm{JH}$, et al. Protein induced by vitamin $\mathrm{K}$ absence or antagonist-II production is a strong predictive marker for extrahepatic metastases in early hepatocellular carcinoma: a prospective evaluation. BMC Cancer. 2011;10:11-43.

[23] Marrero JA, Su GL, Wei W, et al. Des-gamma carboxyprothrombin can differentiate hepatocellular carcinoma from nonmalignant chronic liver disease in american patients. Hepatology. 2003;37:1114-1121.

[24] Mittal A, Gupta SP, Sathian B, et al. Des-gamma-carboxyprothrombin for early identification and prognosis of hepatocellular carcinoma-a case control study from western Nepal. Asian Pac J Cancer Prev. 2012;13:5773-5775.

[25] Huang J, Zeng Y. Current clinical uses of the biomarkers for hepatocellular carcinoma. Drug Discov Ther. 2014;8:98-99.

[26] NCCN Clinical practice in clinical oncology. Hepatobiliary cancers. J Natl Compr Canc Netw. 2009;7:350-391.

[27] Shimizu A, Shiraki K, Ito T, et al. Sequential fluctuation pattern of serum des-gamma-carboxy prothrombin levels detected by highsensitive electrochemiluminescence system as an early predictive marker for hepatocellular carcinoma in patients with cirrhosis. Int J Mol Med. 2002;9:245-250.

[28] Marrero JA, Su GL, Wei W, et al. Des-gamma carboxyprothrombin can differentiate hepatocellular carcinoma from nonmalignant chronic liver disease in American patients. Hepatology. 2003;3:1114-1121.

[29] Jang ES, Jeong SH, Kim JW, et al. Diagnostic performance of alpha-fetoprotein, protein induced by vitamin $\mathrm{K}$ absence, osteopontin, dickkopf-1 and its combinations for hepatocellular carcinoma. PLoS One. 2016;11:e0151069. doi: 10.1371/ journal.pone.0151069.

[30] Ricco G, Cavallone D, Cosma C, et al. Etiology of chronic liver disease and HCC biomarkers. Dig Liver Dis. 2016;48:(Suppl. 1):e52-e53.

[31] Grazi GL, Mazziotti A, Legnani C, et al. The role of tumor markers in the diagnosis of hepatocellular carcinoma, with special reference to the des-gamma-carboxy prothrombin. Liver Transpl Surg. $1995 ; 1: 249-255$. 